Fetal Diagnosis and Therapy

\title{
Is Preterm Delivery Indicated in Fetuses with Gastroschisis and Antenatally Detected Bowel Dilation?
}

\author{
Megan S. Wilson ${ }^{b}$ Mary A. Carroll ${ }^{a}$ b Stephane A. Braun ${ }^{a} \quad$ William F. Walsh $^{a}$ \\ John B. Pietsch ${ }^{a}$ Kelly A. Bennett ${ }^{a, b}$ \\ a Junior League Center for Advanced Maternal Fetal Care, and ${ }^{b}$ Obstetrics and Gynecology, Vanderbilt University \\ School of Medicine, Nashville, Tenn., USA
}

\section{Key Words}

Bowel dilation · Gastroschisis · Ultrasound $\cdot$ Preterm

delivery

\begin{abstract}
Introduction: Due to the controversy surrounding diagnostic ultrasound evaluations and elective preterm delivery of fetuses with gastroschisis, we sought to calculate the predictive value of bowel dilation in fetuses with gastroschisis and evaluate the effect of preterm delivery on neonatal outcomes. Materials and Methods: Ultrasounds and medical records of 103 mother-infant pairs with fetal gastroschisis were reviewed. Eighty-nine pairs met the criteria. Intestinal complications, gestational age at delivery, birth weight, and number of abdominal surgeries were documented. Results: Forty-eight fetuses (54\%) had bowel dilation and 41 (46\%) did not. The positive predictive value of bowel dilation for complicated gastroschisis was $21 \%$. There were 50 (56\%) preterm and 39 (44\%) term deliveries. The mean birth weight was $2,114 \mathrm{~g}(\mathrm{SD}=507)$ and $2,659 \mathrm{~g}(\mathrm{SD}=687), \mathrm{p}=0.001$. For infants delivered preterm, the mean number of postnatal abdominal surgeries was $2.1(\mathrm{SD}=1.1$ ) as compared to 1.3 $(S D=0.5)$ surgical procedures for those infants delivered at term gestation. This was not statistically significant. With respect to hospital stay for each group, the mean length of neonatal intensive care unit admission was 48 days $(S D=33)$
\end{abstract}

in the preterm group and 35 days $(S D=50)$ in the term group, which was not statistically significant. Discussion: Ultrasound-detected bowel dilation was not predictive of important intestinal complications. Our data did not substantiate any benefit for elective preterm delivery of neonates with gastroschisis.

Copyright $\odot 2012$ S. Karger AG, Basel

\section{Introduction}

Gastroschisis is characterized by the herniation of small bowel and other abdominal contents without a covering membrane through a full-thickness abdominal wall defect. Gastroschisis has been estimated to occur in $0.5-1$ per 10,000 births [1]. Recent studies suggest that the prevalence of gastroschisis is rising, with new estimates as high as 5.1 per 10,000 [2]. Overall survival in cases of gastroschisis is very favorable, ranging from $91-97 \%$ [3, 4]. However, survival rates and long-term morbidity are strongly influenced by the presence of bowel pathology at birth. In contrast to uncomplicated cases of gastroschisis, neonates with bowel pathology, such as atresia, infarction, perforation, or volvulus, have a mortality rate up to $28 \%$ [1]. Their postnatal courses may be complicated by prolonged neonatal intensive care unit (NICU) admission, long-term requirement of parenteral-nutrition, mul- 
Fig. 1. Study flow diagram.

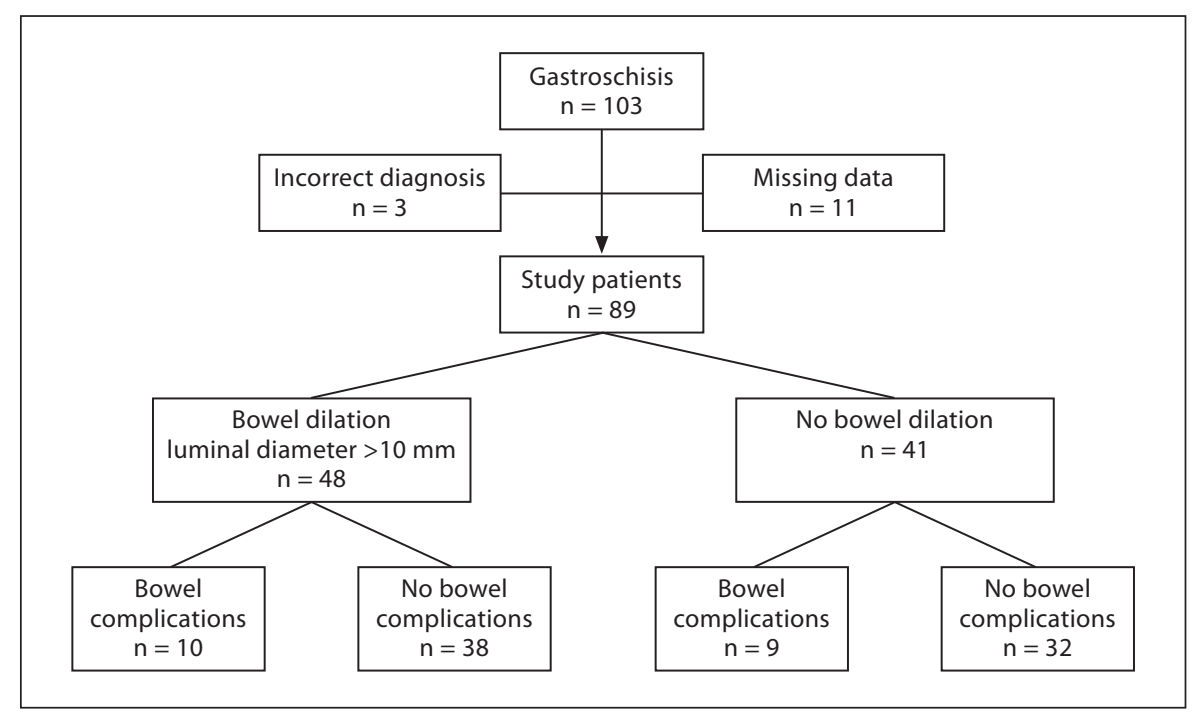

tiple surgical interventions, and development of complications including necrotizing enterocolitis and short bowel syndrome (SBS).

This study was designed to examine whether prenatal identification of bowel dilation was predictive of postnatal bowel pathology and poor neonatal outcomes in a large cohort of fetuses diagnosed with gastroschisis. It also compared features and complications among term neonates with gastroschisis versus their preterm counterparts.

\section{Materials and Methods}

A retrospective review was conducted on all cases of gastroschisis who were evaluated prenatally at the Center for Advanced Maternal Fetal Care (CAMFC) between September 2007 and June 2010. Such cases were identified using the CAMFC fetal anomaly database. The study population included those maternal-fetus pairs with a prenatal diagnosis of gastroschisis, with at least one prenatal ultrasound examination and neonatal care at Vanderbilt University Medical Center (VUMC). Cases with incomplete data or incorrect diagnoses were excluded. Approval was obtained from the institutional review board at VUMC.

Maternal charts were reviewed for maternal age, parity, race, BMI, medications, substance abuse, and antenatal complications. Antenatal complications included bleeding, hypertension, preterm premature rupture of membranes, and infection. Maternal ultrasound reports were reviewed for presence and degree of fetal gastric dilation, intra-abdominal bowel dilation, and extra-abdominal bowel dilation, as well as for presence of oligohydramnios, associated fetal anomalies, and severe intrauterine growth restriction (defined as less than the 3rd percentile). Bowel luminal diameter greater than $10 \mathrm{~mm}$, measured from inner border to inner border, was considered to represent dilation. Prior research in this area has included a wide range of definitions of bowel dilation. Ten millimeters was selected for this analysis as it is an objective value similar to those found to be meaningful in previous research $[5,6]$.

Maternal and neonatal charts were reviewed for delivery characteristics including gestational age at time of delivery, mode of delivery, spontaneous versus induced labor, birth weight, and Apgar scores. (At our institution, patients were followed with twice weekly antepartum fetal testing until spontaneous labor, or scheduled induction at 39 weeks). Neonatal charts were also reviewed for length of NICU admission, number of days of total parenteral nutrition, number of days on mechanical ventilation, number of surgeries, types of surgeries, postnatal intestinal findings, and nursery complications. Postnatal intestinal findings included bowel atresia, malrotation, microcolon, ischemia, bowel resection, and perforation. Nursery complications included sepsis, necrotizing enterocolitis, SBS, requirement of long-term total parenteral nutrition, and neonatal death. The data was reviewed for consistency among data collection sheets and the electronic database prior to final tabulation of results.

Maternal-fetus pairs were grouped according to the presence or absence of bowel dilation on prenatal ultrasound. The neonates were grouped according to preterm or term delivery. Categorical data were compared with a $\chi^{2}$ test. Means and SD were reported and compared using Student's t test; $\mathrm{p}<0.05$ was considered statistically significant. Linear regression with robust standard errors was used to estimate the association between days in the NICU and the presence of any bowel dilation.

\section{Results}

A study flow diagram is shown in figure 1. A total of 103 maternal-fetus pairs were identified with prenatal gastroschisis and evaluated at our center. After exclu- 
Table 1. Maternal demographics and characteristics

\begin{tabular}{llc}
\hline Demographics & Category & Number $(\mathrm{n}=93)$ \\
\hline Age & $16-20$ & $41(44)$ \\
& $21-25$ & $29(31)$ \\
& $26-35$ & $24(25)$ \\
& $>35$ & 0 \\
\hline Race & Caucasian & $76(88)$ \\
& Hispanic & $9(<1)$ \\
& African American & $6(<1)$ \\
& Other & $2(<1)$ \\
\hline Gravidity & Primigravida & $49(53)$ \\
& Multigravida & $44(47)$ \\
\hline Substance abuse & $<20$ & $11(12)$ \\
& $20-25$ & $49(54)$ \\
& $26-30$ & $23(26)$ \\
\hline Maternal complications & Smoker & $7(7)$ \\
& Other & $38(44)$ \\
& Hypertension & $3(13)$ \\
& PROM & $2(2)$ \\
& Infection & $3(3)$ \\
& Other & $6(6)$ \\
\hline
\end{tabular}

Data are presented as $\mathrm{n}(\%)$. sions, 89 maternal-fetus pairs met inclusion criteria and remained in the study.

Table 1 details maternal demographics and characteristics. Median maternal age was 19 years. Fifty-three percent of the women were primiparous. Race and BMI results are presented. Thirty-eight mothers (44\%) self-reported tobacco use during the index pregnancy. There were no cases resulting in intrauterine fetal demise or termination of pregnancy.

Table 2 presents neonatal outcomes associated with the antenatal ultrasound finding of bowel dilation. Fortyeight fetuses (54\%) had ultrasound-documented bowel dilation (intra-, extra-, or both), and 41 (46\%) did not. Average gestational age at delivery and mean birth weight did not differ for the two fetal groups. Similarly, the number of postnatal surgeries did not differ with respect to ultrasound finding of bowel dilation. There was no association between the presence of bowel dilation and days in the NICU (OR: 1.37, 0.57-3.29).

Nineteen of 89 neonates (21\%) had intestinal complications including bowel atresia, malrotation, microcolon, ischemia, bowel resection, short bowel syndrome, and perforation. Of these, only 10 (53\%) had prior identifica-
Table 2. Neonatal outcomes

\begin{tabular}{lccc}
\hline Characteristic & $\begin{array}{l}\text { Dilation } \\
(\mathrm{n}=48)\end{array}$ & $\begin{array}{l}\text { No dilation } \\
(\mathrm{n}=41)\end{array}$ & $\mathrm{p}$ \\
\hline EGA at delivery weeks & $36.2 \pm 1.8$ & $35.9 \pm 2.5$ & NS \\
Birth weight grams & $2,400 \pm 622$ & $2,331 \pm 705$ & NS \\
Length of NICU admission, days & $40 \pm 26$ & $47 \pm 55$ & NS \\
Number of surgeries & $1.8 \pm 1.1$ & $1.7 \pm 1.0$ & NS \\
\hline \multicolumn{2}{c}{ Data are presented as means \pm SD. } & & \\
\hline
\end{tabular}

Table 3. Neonatal outcomes in term and preterm neonates

\begin{tabular}{lccc}
\hline Characteristic & $\begin{array}{l}\text { Preterm } \\
(\mathrm{n}=50)\end{array}$ & $\begin{array}{l}\text { Term } \\
(\mathrm{n}=39)\end{array}$ & $\mathrm{p}$ \\
\hline Birth weight, g & $2,114 \pm 507$ & $2,659 \pm 687$ & 0.001 \\
Number of surgeries & $2.1 \pm 1.1$ & $1.3 \pm 0.5$ & NS \\
Length of NICU admission, days & $48 \pm 33$ & $35 \pm 50$ & NS \\
\hline
\end{tabular}

Data are presented as means $\pm \mathrm{SD}$.

tion of bowel dilation on prenatal ultrasound. The positive predictive value of ultrasound identification of bowel dilation for complicated gastroschisis was $21 \%$.

The postnatal courses of 7 neonates were complicated by SBS. Of the 7 cases, 5 had prenatally identified external bowel dilation. The range of external bowel dilation was 14-26 mm. Three neonates with SBS had prenatally identified internal bowel dilation. The range of internal bowel dilation was $15-21 \mathrm{~mm}$. (One neonate with SBS had both intra- and extra-abdominal bowel dilation).

Table 3 presents neonatal outcomes according to gestational age at delivery. Fifty-six percent of fetuses underwent spontaneous or induced preterm delivery, and $44 \%$ were delivered at term gestation (defined as gestational age greater than 37 weeks). When comparing the difference in mean birth weight for the two groups (preterm delivery vs. term delivery), the difference between the two groups met statistical significance, $2,114 \mathrm{~g}(\mathrm{SD}=$ $507)$ for the preterm delivered cohort and 2,659 $\mathrm{g}(\mathrm{SD}=$ 687 ) for the term delivered cohort, respectively ( $\mathrm{p}=$ $0.001)$.

For infants delivered preterm, the mean number of postnatal abdominal surgeries was $2.1(\mathrm{SD}=1.1)$ as compared to $1.3(\mathrm{SD}=0.5)$ surgical procedures for those infants delivered at term gestation. With respect to hospital 
stay for each group, the mean length of NICU admission was 48 days $(\mathrm{SD}=33)$ in the preterm group and 35 days $(\mathrm{SD}=50)$ in the term group. Neither of these differences met statistical significance.

There was one neonatal death, attributed to sepsis, at 114 days of life. The neonate had a giant gastroschisis with herniation of the liver, managed with attempted staged repair using a silo followed by cadaveric skin graft cover. Her course was complicated by a cutaneous fistula at the base of the gastroschisis, which led to recurrent bleeding which was managed with wound dressings and octreotide infusion. Prenatal ultrasound findings had included both internal and external bowel dilation, ranging 10-20 $\mathrm{mm}$.

\section{Discussion}

Our study sought to evaluate the utility of prenatal ultrasound findings for prediction of neonatal adverse outcome. Additionally, we compared neonatal outcomes of pregnancies complicated by gastroschisis and preterm delivery.

Significant neonatal morbidity is associated with gastroschisis, and prenatal diagnostic findings which would predict those fetuses with the greatest risk have been the subject of numerous reports. There have been conflicting findings with respect to the prognostic ability of these prenatal ultrasound findings to guide the obstetrician and pediatric specialist with respect to timing of delivery and prediction of neonatal morbidity and mortality.

Advances in ultrasound have increased the capability for prenatal diagnosis and surveillance of gastroschisis. At present, approximately $90-98 \%$ of cases of gastroschisis are identified prenatally $[1,9]$. However, some question has been raised whether improvement in prenatal diagnosis of gastroschisis has resulted in improved neonatal outcomes. The ability to affect neonatal outcomes is compromised by the lack of widespread agreement regarding which ultrasound findings are predictive of postnatal bowel pathology, and, in turn, complicated neonatal courses. The presence of bowel dilation as a prognostic factor has been investigated in several studies; however, a consensus has not yet emerged from such work [1,3,10-13]. The absence of strong prognostic factors limits the ability of obstetricians to identify fetuses at risk of complicated neonatal courses, appropriately counsel families, and notify neonatal and pediatric surgical teams accordingly. Additionally, one pitfall of using bowel dilation as an indication for delivery in neonates without pathology is iatrogenic preterm deliv- ery. Preterm neonates with gastroschisis have been reported to have a higher rate of sepsis, longer duration to reach full enteral feedings, and longer hospitalizations than their term counterparts [14]. Commonly cited limitations of the presently available studies addressing ultrasonographic prognostic factors are limited sample size as well as variation in the definition of bowel dilation.

Bowel dilation was not predictive of adverse neonatal outcome in our study cohort. No significant difference was noted with respect to gestational age at delivery, birth weight at delivery, length of NICU stay, or the number of abdominal surgeries required for either abdominal wall closure or for treatment of secondary neonatal complications. Primary closure of the abdominal wall defect was equally likely irrespective of the prenatal ultrasound finding of bowel dilation. Prenatal ultrasound findings were not predictive of postnatal gastrointestinal tract complications.

Our results are in accordance with the findings of $\mathrm{Ba}-$ dillo et al. [12]. His group reported no significant differences between neonates with additional prenatal gastrointestinal abnormalities and neonates with no additional GI abnormalities on prenatal ultrasound with regard to development of complex gastroschisis, gestational age at delivery, or birth weight. However, our results conflict with those of Long et al. [15], who reported a higher death rate as well as a longer period on parenteral nutrition in neonates with prenatally identified bowel dilation. This may be attributable to different definitions of bowel dilation. In our study, $10 \mathrm{~mm}$ was used as a cutoff, whereas the Long group used $25 \mathrm{~mm}$. However, it should be noted that, similar to our study, they did not identify a significant difference in the incidence of intestinal atresia or the number of infants requiring surgery for intestinal failure.

Intestinal bowel wall changes consistent with an increased degree of inflammation have been identified histologically in both human studies and in experimental animal models [16-18]. These inflammatory changes may be mediated by exposure to amniotic fluid constituents. Elective preterm delivery has been proposed as a means of minimizing the harmful exposure of the gastrointestinal tract to the inflammatory effects of amniotic fluid.

In contrast to the work of Moir et al. [19] who reported improved neonatal outcomes with a trial of elective preterm delivery, our data did not substantiate any benefit for the neonates delivered preterm (less than 37 weeks' gestation).

Postoperative return of bowel function as measured by length of days of total parenteral nutrition use was not 
significantly different for the neonates delivered preterm or at term gestation. The mean number of surgeries and length of stay in the NICU was greater in the preterm group, though this finding was not statistically significant.

Published series report an increased rate of stillbirth associated with gastroschisis $[21,22]$. Controlling the risk for intrauterine fetal demise associated with gastroschisis has been a reported rationale in support of elective preterm delivery. Of note, there were no stillbirths in our study cohort of 89 consecutive deliveries. Our protocol for antenatal fetal surveillance of all pregnancies at risk for stillbirth, including those complicated by gastroschisis, entails twice weekly evaluation by alternating nonstress testing and biophysical profile beginning at 32 weeks' gestation. Delivery is recommended regardless of gestational age for nonreassuring fetal status. Whenever feasible, a course of antenatal corticosteroids is administered to fetuses with concern for indicated delivery prior to 34 weeks' gestation. There is a policy of scheduled induction at 39 weeks' gestation for pregnancies affected by gastroschisis.

While this study is limited by its retrospective design, our cohort of 89 mother-infant pairs is the largest number of consecutive cases of gastroschisis in a contemporary series at a single tertiary care center. Over the 32 months of this study interval, there was a uniform treatment plan for prenatal care, ultrasound follow-up, and, postnatally, continuity of both neonatal and pediatric surgical care providers.

We conclude that the antenatal ultrasound finding of bowel dilation is not predictive of neonatal intestinal complications. Furthermore, preterm delivery did not improve neonatal outcomes.

\section{References}

$>1$ Wilson DR, Johnson MP: Congenital abdominal wall defects: an update. Fetal Diagn Ther 2004;19:385-398.

$\checkmark 2$ Mastroiacovo P, Lisi A, Castilla EE: The incidence of gastroschisis: research urgently needs resources. Br Med J 2006;332:250-251.

$>3$ Huh NG, Hirose S, Goldstein RB: Prenatal intraabdominal bowel dilation is associated with postnatal gastrointestinal complications in fetuses with gastroschisis. Am J Obstet Gynecol 2010;202:396.e1-e6.

4 Molik KA, Gingalewski CA, West KW, Rescorla FJ, Scherer LR, Engum SA, Grosfeld JL: Gastroschisis: a plea for risk categorization. J Pediatr Surg 2001;36:51-55.

5 Babcook CJ, Hedrick MH, Goldstein RB, Callen PW, Harrison MR, Adzick NS, Filly RA: Gastroschisis: can sonography of the fetal bowel accurately predict postnatal outcome? J Ultrasound Med 1994;13:701-706.

$\checkmark 6$ Piper HG, Jaksic T: The impact of prenatal bowel dilation on clinical outcomes in neonates with gastroschisis. J Pediatr Surg 2006; 41:897-900.

7 Jeejeebhoy KN: Short bowel syndrome: a nutritional and medical approach. CMAJ 2002; 166:1297-1302.

$>8$ Sala D, Chomto S, Hill S: Long-term outcomes of short bowel syndrome requiring long-term/home intravenous nutrition compared in children with gastroschisis and those with volvulus. Transplant Proc 2010; 42:5-8.
9 Garne E, Loane M, Dolk H, EUROCAT Working Group: Gastrointestinal malformations: impact of prenatal diagnosis on gestational age at birth. Paediatr Perinat Epidemiol 2007;21:370-375.

10 Japaraj RP, Hockey R, Chan FY: Gastroschisis: can prenatal sonography predict neonatal outcome? Ultrasound Obstet Gynecol 2003;21:329-333.

-11 Alsulyman OM, Monteiro H, Ouzounian JG, Barton L, Songster GS, Kovacs BW: Clinical significance of prenatal ultrasonographic intestinal dilatation in fetuses with gastroschisis. Am J Obstet Gynecol 1996;175;982-984.

12 Badillo AT, Hedrick HL, Wilson RD, et al: Prenatal ultrasonographic gastrointestinal abnormalities, in fetuses with gastroschisis do not correlate with postnatal outcomes. J Pediatr Surg 2008;43:647-653.

13 Nick AM, Bruner JP, Moses R, Yang EY, Scott TA. Second-trimester intra-abdominal bowel dilation in fetuses with gastroschisis predicts neonatal bowel atresia. Ultrasound $\mathrm{Ob}$ stet Gynecol 2006;28:821-825.

14 Maramreddy H, Fisher J, Slim M, Lagamma EF, Parvez B: Delivery of gastroschisis patients before 37 weeks of gestation is associated with increased morbidities. J Pediatr Surg 2009;44:1360-1366.

15 Long AM, Court J, Morabiro A, Gilham JC: Antenatal diagnosis of bowel dilation in gastroschisis is predictive of poor postnatal outcome. J Pediatr Surg 2011;46:1070-1075.

16 Guibourdenche J, Berrebi D, Vuillard E, de Lagausie P, Aigrain Y, Oury JF, Luton D: Biochemical investigations for bowel inflammation in gastroschisis. Pediatr Res 2006;60: 565-568.
17 Burc L, Volumenie JL, de Lagausie P, Guibourdenche J, Oury JF, Vuillard E, Sibony O, Blot P, Saizou C, Luton D: Amniotic fluid inflammatory proteins and digestive compounds profile in fetuses with gastroschisis undergoing amnioexchange. BJOG 2004; 111:292-297.

-18 Langer J, Longaker MT, Crombleholme TM, Bond SJ, Finkbeiner WE, Rudolph CA, Verrier ED, Harrison M: Etiology of intestinal damage in gastroschisis. I: effects of amniotic fluid exposure and bowel constriction in a fetal lamb model. J Pediatr Surg 1989;24: 992-997.

19 Moir C, Ramsey PS, Ogburn PL, Johnson RV, Ramin KD: A prospective trial of elective preterm delivery for fetal gastroschisis. Am J Perinatol 2004:21:289-294.

20 Huang J, Kurkchubasche AG, Carr SR, Wesselhoeft CW Jr, Tracy TF Jr, Luks FL: Benefits of term delivery in infants with antenatally diagnosed gastroschisis. Obstet Gynecol 1999;94:112-116.

-21 Santiago-Munoz, McIntire DD, Barber RG, Megison SM, Twickler DM, Dashe JS: Outcomes of pregnancies with fetal gastroschisis. Obstet Gynecol 2007;110:663-668.

22 David AL, Tan A, Curry J: Gastroschisis: sonographic diagnosis, associations, management and outcome. Prenat Diagn 2008;28: 633-644. 